\title{
EDITORIAL
}

\section{INDICADORES DE CALIDAD EN EL CUIDADO DE ENFERMERÍA: ESTRATEGIA PARA LA COBERTURA UNIVERSAL DE SALUD}

\author{
QUALITY INDICATORS IN THE NURSING CARE: \\ STRATEGY FOR THE UNIVERSAL HEALTH COVERAGE \\ Dra. Sara Guerrero Núñez \\ Departamento de Enfermería, Facultad Ciencias de la Salud, \\ Universidad de Atacama. Chile.
}

Email: sara.guerrero@uda.cl

La Cobertura Universal de Salud es una política pública, definida como la "capacidad del sistema de salud para responder a las necesidades en salud de la población, lo cual incluye la disponibilidad de infraestructura, recursos humanos, tecnologías de la salud (incluyendo medicamentos) y financiamiento" (1). Los Estados miembros de la Organización Mundial de la Salud asumieron en 2005 el compromiso de alcanzarla, convencidos que "todas las personas deberían tener acceso a los servicios de salud que necesitan sin correr el riesgo de ruina económica o empobrecimiento" (2). El plan estratégico de la Organización Panamericana de la Salud 2014-2019 indica que la Cobertura Universal de Salud y los Determinantes Sociales en Salud son fundamentales en un sistema que desea impactar equitativamente en la salud de la población.

Es responsabilidad de los gobiernos decidir "qué servicios de salud son necesarios, y cómo asegurarse de que estén universalmente disponibles y sean asequibles, eficaces y de buena calidad" (2). Por ello, es primordial evaluar el progreso de esta política de salud, además de definir estrategias específicas que permitan medirla. Las investigaciones contribuyen en esto, otorgando soluciones a problemas de salud, y definiendo estrategias eficaces para mejorar la salud y equidad sanitaria (3).

Para responder a una Cobertura Universal de Salud en el ámbito del cuidado, es necesario que enfermería integre las investigaciones multidisciplinarias dirigidas a mejorar dicha cobertura. Su desafío radica en la producción de conocimiento en el ámbito del cuidado para una Cobertura Universal de Salud, y en la implementación de estrategias o medidas que emerjan de ese conocimiento. Como disciplina profesional, enfermería fortalece los sistemas de salud al contribuir con el acceso 
a una salud integral, y al aportar datos científicos para la innovación y eficacia de los sistemas (4).

La calidad es una característica esencial de la Cobertura Universal de Salud (5). En consecuencia, el cuidado para una Cobertura Universal de Salud incorporará la calidad como eje central del quehacer de enfermería, estableciendo un sistema de gestión de la calidad del cuidado. Para el mejoramiento continuo de la calidad asistencial, los profesionales enfermeros deben identificar factores intervinientes, implementar acciones, y diseñar instrumentos para una evaluación sistemática de la calidad del cuidado (6). Es preciso que los enfermeros identifiquen el "conjunto de datos mínimos indispensables para la descripción y monitorización de su práctica" (6). Por esta razón, los indicadores de calidad en el cuidado de enfermería son herramientas esenciales y útiles para el mejoramiento continuo del cuidado y de la atención de salud, especialmente en el contexto de problemáticas de salud pública con importancia local, nacional, e internacional.

Son diversos los desafíos para evaluar el progreso de la Cobertura Universal de Salud en el ámbito del cuidado de enfermería, pero un importante avance es la definición de un marco conceptual de monitoreo (7), y la definición y validación de indicadores (8), especialmente indicadores de calidad, los cuales respondan a necesidades de cuidado en la población. Para esto enfermería requerirá sistematizar el conocimiento producido en los diversos contextos sanitarios, políticos y sociales, a nivel nacional e internacional.

Los indicadores de calidad en el cuidado de enfermería corresponden a una eficiente estrategia para alcanzar la Cobertura Universal de Salud, permitiendo una evaluación de la estructura, proceso, resultado e impacto del cuidado. Estos indicadores informan sobre las condiciones mínimas que deben observarse en la práctica de enfermería, necesarias para el acceso y cobertura universal de las necesidades de salud. En consecuencia, los(as) enfermeros(as) disponen de herramientas para evaluar e intervenir en Cobertura Universal de Salud, cuando definen y validan indicadores de calidad en las diversas dimensiones de esta cobertura. Algunas de las dimensiones corresponden a cobertura poblacional, cobertura de prestación de servicios, protección financiera, determinantes sociales de la salud, equidad en salud y carga de enfermedad (8). Aunque los países han fortalecido sus sistemas de salud para implementar la política pública de Cobertura Universal de Salud, enfermería debe integrar los esfuerzos realizados, desarrollando investigaciones para la construcción de un marco conceptual de monitoreo, y la implementación de indicadores de calidad, principalmente, en enfermedades de mayor prevalencia poblacional. 


\section{REFERENCIAS}

1. Organización Mundial de la Salud (OMS), Organización Panamericana de la Salud (OPS). Estrategia para el acceso universal a la salud y la cobertura universal de salud. 53 $3^{\text {er }}$ Consejo Directivo, 66 Sesión del Comité Regional de la OMS para las Américas, del 29 de septiembre al 3 de octubre de 2014. Documento oficial CD53/5 [Internet]. Washington, D.C: OMS-OPS; 2014 [citado 2 abr 2018]. 26 p. Disponible en: http://www.paho.org/HQ/index. php?option=com_content\&view=article\&id=9774\&Itemid=41062\&lang=es

2. Organización Mundial de la Salud (OMS). Investigaciones para una cobertura sanitaria universal, Informe sobre la salud en el mundo 2013 [Internet]. Ginebra: OMS; 2013 [citado 30 mar 2018]. 160 p. Disponible en: http://www.who. int/whr/2013/report/es/

3. Organización Mundial de la Salud (OMS). Función y responsabilidades de la OMS en las investigaciones sanitarias. 63a Asamblea Mundial de la Salud. WHA63.21 [Internet]. Washington, D.C: OMS; 2010 [citado 4 abr 2018]. 28 p. Disponible en: http://apps.who.int/gb/ebwha/pdf_files/WHA63/A63_22-sp. pdf

4. Organización Mundial de la Salud (OMS). Fortalecimiento de la enfermería y la partería. 64a Asamblea Mundial de la Salud. WHA64.7 [Internet]. Washington, D.C: OMS; 2011 [citado 9 abr 2018]. 4 p. Disponible en: http://apps.who.int/ iris/handle/10665/5427

5. Storr J, Kilpatrick C, Allegranzi B, Syed S. Redefining infection prevention and control in the new era of quality universal health coverage. J Res Nurs. 2016; 21(1): 39-52.

6. Gabriel C, Da Costa M, Rossi F, Bernardes A, Miguelaci T, Prado M. Utilización de indicadores de desempeño en servicio de enfermería de hospital público. Rev Lat Am Enfermagem. 2011; 19(5): 1247-1254.

7. Báscolo E, Houghton N, Del Riego A. Construcción de un marco de monitoreo para la salud universal. Rev Panam Salud Publica. 2018; 42: e81.

8. Pinzón C, Chapman E, Panisset U, Arredondo A, Fitzgerald J, Reveiz L. Disponibilidad de indicadores para el seguimiento del alcance de la "Salud Universal" en América Latina y el Caribe. Rev Panam Salud Publica. 2016; 39(6): 330-340. 